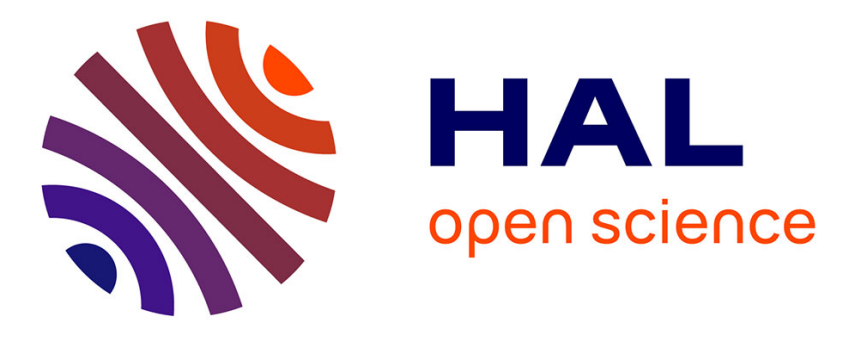

\title{
Méthodologie des recommandations pour la pratique clinique concernant la prise en charge des pertes de grossesse
}

\author{
X. Deffieux, C. Huchon, Amélie Delabaere, V. Lavoue, S. Nedellec, V. Gallot, \\ P. Capmas, G. Beucher, X. Carcopino, François Vialard, et al.
}

\section{To cite this version:}

X. Deffieux, C. Huchon, Amélie Delabaere, V. Lavoue, S. Nedellec, et al.. Méthodologie des recommandations pour la pratique clinique concernant la prise en charge des pertes de grossesse. Journal de Gynécologie Obstétrique et Biologie de la Reproduction, 2014, 43 (10), pp.753-755. 10.1016/j.jgyn.2014.09.009 . hal-02077430

\section{HAL Id: hal-02077430 https://hal.science/hal-02077430}

Submitted on 29 Nov 2019

HAL is a multi-disciplinary open access archive for the deposit and dissemination of scientific research documents, whether they are published or not. The documents may come from teaching and research institutions in France or abroad, or from public or private research centers.
L'archive ouverte pluridisciplinaire HAL, est destinée au dépôt et à la diffusion de documents scientifiques de niveau recherche, publiés ou non, émanant des établissements d'enseignement et de recherche français ou étrangers, des laboratoires publics ou privés. 


\title{
PERTES DE GROSSESSE
}

\section{Méthodologie des recommandations pour la pratique clinique concernant la prise en charge des pertes de grossesse}

\section{Methods used to provide guidelines for clinical practice concerning the management of pregnancy losses}

\author{
X. Deffieux ${ }^{a, *}$, C. Huchon ${ }^{b, c}$, A. Delabaere ${ }^{d, e}$, V. Lavoue ${ }^{f}$, \\ S. Nedellec ${ }^{a}$, V. Gallot ${ }^{a}$, P. Capmas ${ }^{\mathrm{g}, \mathrm{h}}$, G. Beucher ${ }^{\mathrm{i}}$, \\ X. Carcopino ${ }^{j, k}$, F. Vialard ${ }^{1, m}$, J. Nizard $^{\mathrm{n}, \mathrm{o}}$, T. $^{\text {Q }}$ Quibel $^{\mathrm{b}}$, \\ N. Costedoat-Chalumeau ${ }^{p}, G$. Legendre ${ }^{q}$, D. Lemery $^{\mathrm{d}, \mathrm{r}}$
}

\footnotetext{
a Service de gynécologie-obstétrique et médecine de la reproduction, hôpital Antoine-Béclère, AP-HP, 157, rue de la Porte-de-Trivaux, 92140 Clamart, France

b Service de gynécologie-obstétrique, CHI Poissy-Saint-Germain-en-Laye, 10, rue du Champ-Gaillard, 78300 Poissy, France

' EA 7285, risques cliniques et sécurité en santé des femmes, université Versailles-Saint-Quentin-en-Yvelines, 78000 Versailles, France

d Pôle de gynécologie-obstétrique reproduction humaine, CHU Estaing, 1, place Lucie-Aubrac, 63003 Clermont-Ferrand cedex 1, France

e EA7281-R2D2, faculté de médecine, université d'Auvergne, 28, place Henri-Dunant, 63000 Clermont-Ferrand, France

f Inserm U1085, service de gynécologie, hôpital Sud, CHU de Rennes, université de Rennes-1, 16, boulevard de Bulgarie, 35000 Rennes, France

's Service de gynécologie-obstétrique, hôpital Bicêtre, GHU Sud, AP-HP, 78, rue du Général-Leclerc, 94276 Le Kremlin-Bicêtre, France

$\mathrm{h}$ Inserm, centre de recherche en épidémiologie et santé des populations (CESP), U1018, faculté de médecine, université Paris-Sud, 94276 Le Kremlin-Bicêtre, France

i Service de gynécologie-obstétrique et médecine de la reproduction, CHU de Caen, avenue Côte-de-Nacre, 14033 Caen cedex 9, France

j Service de gynécologie-obstétrique, hôpital Nord, AP-HM, chemin des Bourrely, 13915 Marseille cedex 20, France

${ }^{k}$ Faculté de médecine, université d'Aix-Marseille université (AMU), 27, boulevard Jean-Moulin, 13385 Marseille cedex 05, France

' Unité de cytogénétique, CHI Poissy-Saint-Germain, 10, rue du Champ-Gaillard, 78300 Poissy, France
}

* Auteur correspondant.

Adresse e-mail : xavier.deffieux@abc.aphp.fr (X. Deffieux). 
m EA 2493, UFR des sciences de la santé Simone-Veil, université Versailles-Saint-Quentin-en-Yvelines, 78000

Versailles, France

n Service de gynécologie-obstétrique, groupe hospitalier Pitié-Salpêtrière, AP-HP, 47-83, boulevard de

l'Hôpital, 75013 Paris, France

- Inserm U1150, CNRS UMR 7222, UPMC université Paris 06, Sorbonne universités, 75005 Paris, France

$\mathrm{P}$ Service de médecine interne, centre de référence maladies auto-immunes et maladies systémiques rares,

hôpital Cochin, 27, rue du Faubourg-Saint-Jacques, 75013 Paris, France

q Service de gynécologie-obstétrique, CHU Angers, 4, rue Larrey, 49033 Angers cedex 01, France

r EA 4681 PEPRADE, faculté de médecine, université d'Auvergne, 28, place Henri-Dunant, 63000

Clermont-Ferrand, France

\section{Introduction}

L'objectif de ces recommandations pour la pratique clinique (RPC) du Collège national des gynécologues et obstétriciens français (CNGOF) était d'améliorer les pratiques des professionnels de santé (gynécologues, gynécologues obstétriciens, chirurgiens) concernant les pertes de grossesse afin d'en optimiser le diagnostic et le traitement.

\section{Matériel et méthode}

La Haute Autorité de santé (HAS) a défini un certain nombre de principes méthodologiques que nous avons adoptés pour élaborer ces RPC $[1,2]$. Notre méthodologie répond aux critères de qualité recensés dans la grille d'évaluation de la qualité des RPC (dérivée de la grille AGREE II) [3].

Le promoteur de cette RPC était le CNGOF dont le bureau constitue le comité d'organisation. La charge de ce bureau, en lien avec Gynerisq est de définir les questions posées de façon précise et de définir ses limites, de désigner les membres du groupe de travail et de relire et valider les conclusions du groupe.

Le CNGOF a posé un certain nombre de questions au groupe de travail: Quelle doivent être les termes utilisés pour définir les différentes pertes de grossesse? Quelle est la prévalence des différents types de pertes de grossesse? Quelle est la stratégie diagnostique et thérapeutique à adopter devant des symptômes faisant craindre une perte de fausse couche au premier trimestre? Quel est le bilan étiologique à prescrire devant des fausses couches à répétition? Y a-t-il des métiers exposés aux fausses couches, pour lesquels une prévention pourrait être utile? Comment prévenir le risque de fausse couche en cas de syndrome des antiphospholipides, de thrombophilie, de diabète, de dysthyroïdie, d'obésité? Comment prendre en charge une fausse couche tardive? Quelles sont les indications validées de cerclage? Qu'est-ce qu'une béance cervico-isthmique? Comment prendre en charge une femme présentant une poche des eaux dans le vagin avant 24SA? Quel bilan étiologique prescrire après une fausse couche tardive? Comment prévenir les complications des fausses couches (rétention, endométrite)? Quels sont les résultats des différentes stratégies (expectative, misoprostol (per os, vaginal, rectal), syntocinon, methergin, mifépristone, mifépristone + misoprostol, traitement, aspiration) en cas de fausse couche ou de grossesse arrêtée au premier trimestre? Quel est le bilan étiologique à prescrire devant une mort fœetale in utero? Y a-t-il des causes psychologiques aux fausses couches (rôle du stress, de la dépression)? Quelle doit être la prise en charge psychologique d'une fausse couche et d'une mort fœtale in utéro?

Les critères de sélection des preuves étaient clairement décrits. Seules les méta-analyses, les essais randomisés, les études comparatives et les grandes cohortes ont été retenues pour les recommandations de grade $\mathrm{A}$ ou $\mathrm{B}$. Ce travail s'appuie sur une revue exhaustive de la littérature de 1975 à juillet 2014. Bases de données consultées: (PubMed, Medline, Cochrane library, Cochrane database of systemactic reviews, EMBASE, US National Guideline Clearinghouse, guidelines, RCOG, ACOG) concernant les méta-analyses, essais randomisés, registres, revues de la littérature, études contrôlées et grandes études non contrôlées, publiés sur le sujet jusqu'en juillet 2014 (en langue française ou anglo-saxonne). Les études animales ou anatomiques ont été exclues. Les experts rédacteurs ont synthétisé la littérature pour chacune des questions abordées et les recommandations ont été élaborées par un groupe de travail multidisciplinaire, puis ces recommandations ont été relues et amendées par un groupe de relecteurs experts (gynécologues, sages-femmes, chirurgiens gynécologues, gynécologues obstétriciens, internistes, généticiens).

Mots clés utilisés pour la recherche bibliographique : biochemical pregnancy; miscarriage; fetal loss; pregnancy loss; missed miscarriage; early miscarriage; retained products of conception; incomplete miscarriage; repeat miscarriage; recurrent pregnancy loss; cerclage; late miscarriage; threatened miscarriage; shortening cervix; opening cervix; fetal death; intrauterine fetal demise.

En suivant les recommandations 2010 de la HAS $[1,2]$, l'argumentaire scientifique comprenait pour chaque question un chapitre incluant une synthèse critique et concise de la littérature retenue, un texte référencé avec mention des niveaux de preuve (NP) et des tableaux de synthèse, les points d'hétérogénéité ou de divergence de pratique ainsi qu'une conclusion avec les recommandations, gradées et validées à l'issue de la phase de finalisation (relecture). 
Les propositions de recommandations et l'argumentaire complet ont été envoyés à 47 relecteurs début juillet 2014 . Fin août 2014, après analyse et discussion des commentaires du groupe de lecture, les recommandations initiales ont été éventuellement modifiées par le groupe de travail.

En cas de recommandation fondée sur un niveau de preuve élevé (grade $A$ ou $B$ ), le groupe de travail a pris en compte les commentaires pertinents pour améliorer la forme, et a éventuellement apporté des modifications sur le fond, s'il y avait lieu, en fonction des données supplémentaires fournies (article ou données de pratique) avec parfois une modification du grade de la recommandation si nécessaire. Pour les recommandations fondées sur un faible niveau de preuve (grade $\mathrm{C}$ ), lorsque le groupe de relecture confirmait nettement le caractère approprié de la recommandation, la recommandation était conservée et les commentaires pertinents étaient pris en compte pour améliorer la forme. Lorsque le groupe de relecture était plus largement indécis ou en désaccord avec la recommandation initiale, le groupe de travail a discuté de la pertinence des commentaires et modifié s'il y avait lieu la recommandation. Quand les débats en réunion ont fait apparaître des divergences de points de vue, un vote en séance du groupe de travail a confirmé l'abandon ou la formulation finale de la recommandation modifiée.

Patientes auxquelles la RPC doit s'appliquer:

- Concernant les pertes de grossesse, ces RPC s'appliquent aux femmes de 15 ans et plus, prises en charge pour une perte de grossesse ou des symptômes pouvant faire évoquer un risque de perte de perte de grossesse (saignements, douleurs ou contractions).

- Ces RPC excluent les patientes sous anticoagulants pour lesquelles les stratégies peuvent être différentes.

Les utilisateurs cibles de la RPC sont les gynécologues médicaux, les sages-femmes, les médecins généralistes et les gynécologues obstétriciens prenant en charge des femmes enceintes ou en âge de procréer.

Une procédure de réactualisation de la RPC est prévue dans 5 à 10 ans.

La clarté et la présentation ont été soignées. Les recommandations clés seront facilement identifiables sous la forme d'un résumé (synthèse) rappelant les recommandations de grade élevé. La RPC sera accompagnée d'outils permettant son application: mise en ligne en accès libre sur le site du CNGOF des recommandations.

La rédaction de la RPC était indépendante des organismes de financement. Les conflits d'intérêt des membres du groupe ayant élaboré la RPC sont documentés.

\section{Discussion}

Une des principales difficultés rencontrées était l'absence de définitions internationalement reconnues. Ainsi, d'une publication à l'autre, les groupes de «fausse couche» ou de «mort fœtale in utéro» étaient souvent très hétérogènes, en particulier concernant l'âge gestationnel auquel la perte de grossesse était survenue. Ceci a rendu l'analyse de la littérature très difficile par moments. Plusieurs sociétés savantes anglo-saxonnes (American College of Obstetricians and Gynecologists [ACOG] ; American Society of Reproductive Medecine [ASRM] ; Queensland Maternity and Neonatal Clinical Guidelines Program [Australia and New Zeland]; National Institute for Health and Clinical Excellence [NICE]; Royal College of Obstetrics and Gynaecology [RCOG]) ont publié des définitions et recommandations de terminologie concernant l'évaluation de l'évolutivité du début de grossesse et les pertes de grossesse, mais ces définitions publiées ne sont pas consensuelles d'une société à l'autre et aucun auteur francophone n'a participé à leur élaboration. Il n'était donc pas possible de proposer une simple traduction française des définitions anglo-saxonnes existantes. Le Collège national des gynécologues et obstétriciens français (CNGOF) a donc réuni un groupe de travail multidisciplinaire afin de définir une terminologie française commune concernant les définitions portant sur l'état d'évolution d'une grossesse et la définition des différents types de pertes d'une grossesse. Une méthode inspirée des «recommandations par consensus formalisé » de la HAS de 2010 a été choisie pour cette partie de nos recommandations, car les conditions suivantes étaient remplies: absence ou insuffisance de littérature de fort niveau de preuve et absence de terminologie internationale consensuelle.

Bien entendu, l'élaboration de ces recommandations n'est pas censée répondre à toutes les situations cliniques, mais au plus grand nombre. De plus, elles sont appelées à évoluer car beaucoup d'essais sont actuellement menés sur cette thématique.

\section{Conclusion}

Ces recommandations devraient aider tous les professionnels de santé à mieux prendre en charge les femmes posant le problème d'une perte de grossesse.

\section{Références}

[1] Rédaction de l'argumentaire scientifique-Élaboration de recommandations de bonne pratique et des recommandations. Fiche méthodologique de la HAS; 2010.

[2] Méthode recommandations pour la pratique cliniqueÉlaboration de recommandations de bonne pratique. Fiche méthodologique de la HAS; 2010.

[3] Grille d'évaluation de la qualité des recommandations de bonne pratique (dérivée de la grille AGREE II). Fiche méthodologique de la HAS; 2011. 